\title{
Water Cherenkov Detector of the JUNO Veto System
}

\author{
R. G. Wang*, C. G. Yang, H. Q. Lu, J. C. Liu, P. Zhang, C. Guo, Y. P. Zhang, on behalf \\ of the JUNO Collaboration \\ Institute of High Energy Physics, Chinese Academe of Sciences, Beijing, China \\ E-mail: wangrgeihep.ac.cn
}

\begin{abstract}
The Jiangmen Underground Neutrino Observatory (JUNO) is a 20 kton liquid scintillator detector with primary physics goal of neutrino mass hierarchy determination and precise measurement of the neutrino oscillation parameters, as well as the measurement of solar neutrinos, geo-neutrinos, supernova neutrinos and the diffuse supernova neutrinos. The detector will be built in a 700 $\mathrm{m}$ deep underground laboratory. A multi-veto system will be built for cosmic muon detection and background reduction. The central detector will be submerged in the ultra pure water pool instrumented with about 2000 MCP-PMTs (20 inches) to serve as an active water Cherenkov detector for muon tagging. Both the pool walls and the central detector external surface will be coated with Tyvek reflector to increase the light collection efficiency. The muon detection efficiency will be $>95 \%$ for water Cherenkov detector. With this veto system, the cosmic muon induced fast neutron background can be reduced to the level of $\sim 0.1 /$ day.
\end{abstract}

European Physical Society Conference on High Energy Physics - EPS-HEP2019 -

10-17 July, 2019

Ghent, Belgium

\footnotetext{
* Speaker.
} 


\section{The JUNO experiment}

The JUNO experiment is located in the south of China (Jiangmen city, Guangdong province) and at about $53 \mathrm{~km}$ from two nuclear power plants Taishan and Yiangjian, which will provide antineutrino flux for JUNO with total thermal power of $36.8 \mathrm{GW}$ (the actual power will be $26.6 \mathrm{GW}$ in 2021). The detector is built underground with an overburden of $650 \mathrm{~m}$ of granite rock to reduce the amount of cosmogenic muons as background source. It is consists of a 20 kton liquid scintillator (LS) with larger than 75\% PMT photocathode coverage to achieve 3\%/ $\sqrt{E(\mathrm{MeV})}$ energy resolution. The JUNO detector includes the Central Detector (CD), veto detector and other systems [ㅁ, ㅁ], shown in figure 1. The veto system used for muon induced background study and reduction consists of a Water Cherenkov Detector (WCD), a Top Tracker (TT) and other subsystems. This paper will mainly introduce design and construction of WCD and other subsystems.

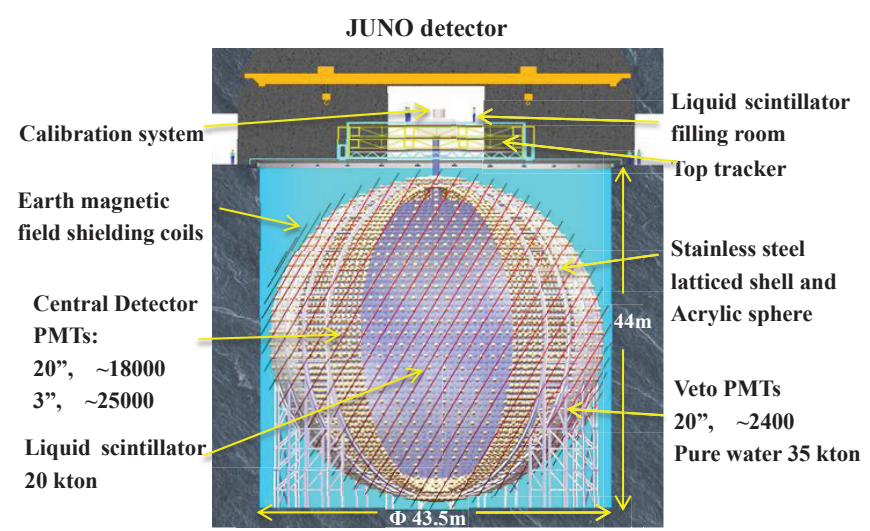

Figure 1: A schematic view of the JUNO detector.

\section{Water Cherenkov Detector}

The WCD is a cylindrically shaped pool filled with purified water and instrumented with PMTs surrounding the CD. The water pool has a diameter of $43.5 \mathrm{~m}$ and a depth of $44 \mathrm{~m}$ lined with high density polyethylene plate ( HDPE). The PMT arrangement is optimized for high muon detection efficiency and good track resolution. The surface of this detector is covered by Tyvek as reflecting and diffusing sheets to increase the light detection. In order to achieve the designed performance, a water circulation/purification system with radon removal function will be built.

\subsection{Water pool liner}

The pool's inner surface will be lined with HDPE plate to separate inner pure water from outer rock. It also prevents radon diffusion and water seeping from surrounding rock into the pool. HDPE has good corrosion resistance, high strength, good ductility, and compatibility with pure water. Figure 2 shows two kinds of HDPE liner used in JUNO pool, one is called StudLiner with nails for the pool wall and another is smooth liner without nails for the pool bottom and the cable trench (also see Figure 5). The StudLiner with thickness of $5 \mathrm{~mm}$ will be installed at the same time as pool pouring. The smooth liner is $2 \mathrm{~mm}$ and $3 \mathrm{~mm}$ thick, they will be installed after the central detector will be built. 


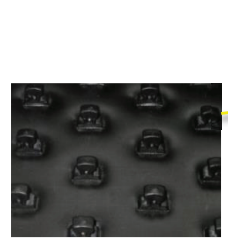

On wall

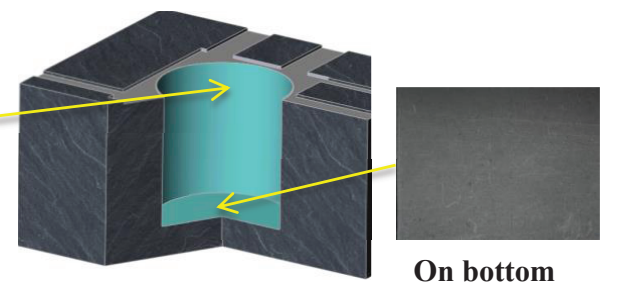

Figure 2: HDPE liner. Left: the StudLiner. Middle: the pool. Right: the smooth liner.

\subsection{Veto PMTs}

Veto PMTs are placed in the water pool to tag cosmic ray muons by detecting water Cherenkov light of muons traversing the JUNO detector. Total about 200020 inch veto PMTs are uniformly distributed on the outer surface of the the CD stainless steel frame, $44 \%$ on the top and $56 \%$ on the bottom halves, respectively. In order to satisfy the requirement of electronics box matching and installation, the PMT distribution region is divided 10 pieces according to longitude. Each piece is as a veto PMT trigger unit. In this case, muon tagging efficiency is expected to be $>95 \%$, fast neutron background can be maintained at 0.1 event/day. PMTs distribution and trigger scheme are showed in Figure 3.
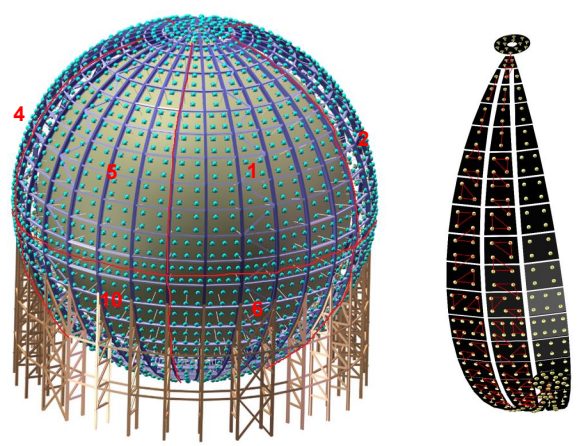

Figure 3: Left: PMTs distribution on the outer surface of the CD stainless steel frame. Right: a trigger unit divided for a trigger scheme.

\subsection{Tyvek reflection film}

In order to collect enough photons without using a large number of veto PMTs, the inner surface of the pool (including wall, bottom and top) and the outer surface of the central detector will be covered with high reflectivity Tyvek film. This Tyvek reflective material, as proved in the Daya Bay experiment, has the merits of high reflectivity ( $>95 \%$ when wavelength longer than 300 $\mathrm{nm}$, seeing Figure 4) and stable performance [[]]. Since Tyvek could be combined with polyethylene film to form a multi-layer film, actual Tyvek films are often multilayers of appropriate thickness. Because two pieces of Tyvek can be easily welded together, we can make different size reflectors to meet large detector installation. Based on the experience of Daya Bay experiment and more mature production technology, the Tyvek reflector made for JUNO is expected better performance. 

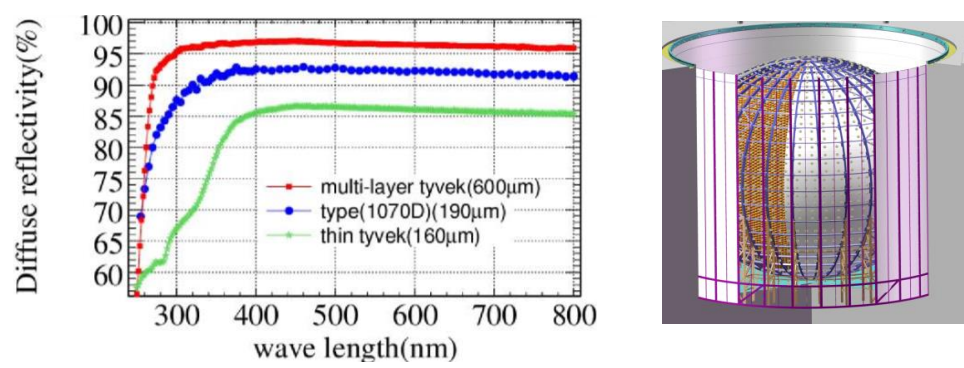

Figure 4: Left: reflectivity of different Tyveks . Right: effect drawing of Tyvek.

\subsection{Cover and cable trench}

The Daya Bay experiment has successfully used a black rubber cloth served as the pool cover which shields the pool from the external air and light. In JUNO we still adopt this cover technique for pool seal. Because the JUNO pool is very large with a 40-meter span, it is difficult to install such a large black rubber cloth on top of the pool. Therefore, our preliminary design scheme is: first, install sliding bars and sliders on both sides of the TT bridge for cover support; second, the cover is divided into two pieces which will be connected together with a seal zipper after installation, many ropes of a certain length with a ring at one en are evenly fixed to the cover cloth for suspension; third, slowly and gradually pull and unfold the each part of rolled cover from one side of the pool to another side, meanwhile put rope rings on the sliding bars, then zip up two pieces of cover; finally, the cover edge is fixed on the outside edge of the pool and inside the cable trench. Figure 5 simply illustrates the above installation process. The cover edge and cable in the cable trench will be submerged in water in order to maintain good tightness of the pool.
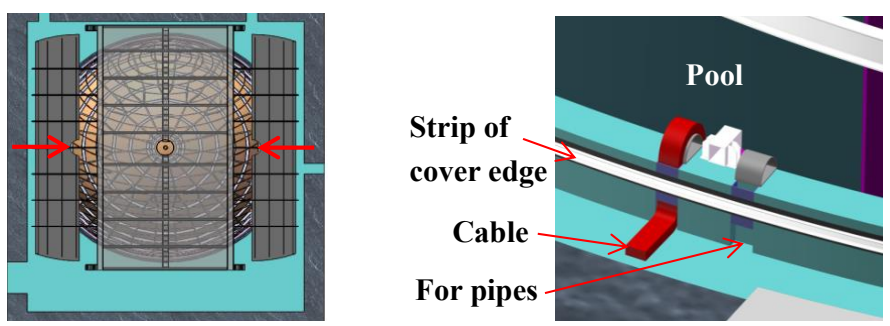

Figure 5: Left: cover installation. Right: cable trench.

\subsection{Water system}

It is necessary to build a reliable production, purification and circulation system for JUNO detector operation. This pure water system has three functions: one is to ensure pool water high transparency; another is to keep the overall central detector (especially the acrylic sphere) temperature stable which is critical for the entire experiment; third is removal of Radon from water.

The JUNO water system consists of the ultra-pure water production system and the circulation/purification system. To reduce waste water treatment as much as possible, the water production system is divided into two parts, one is on the surface ground and another is in underground experiment hall. Many small particles of insoluble material and ions could be removed from city water 
with a filter and reverse osmosis (RO) system on the ground. The pre-purified water then pipes to underground for further purification, including RO again, total organic carbon(TOC) removal, electrodeionization(EDI), resin, ultraviolet, microfilter, multiple degasser. The whole process is shown in Figure 6. In the end the resistance is greater than $16 \mathrm{M} \mathrm{Ohm}$ and the radon concentration is less than $0.2 \mathrm{~Bq} / \mathrm{m}^{3}$. The simulation shows that water inlet from the upper and lower poles and outlet at near equator will effectivelly maintain CD temperature and prevent Radon diffusion to the CD. Figure 7 shows the sketch of water path and temperature distribution.

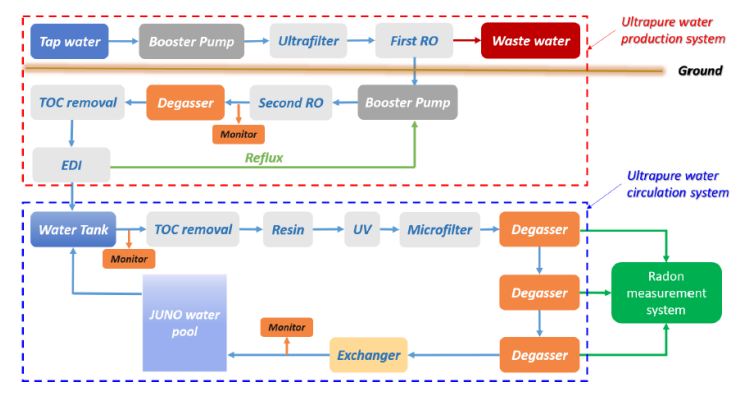

Figure 6: The flow chart of JUNO pure water system
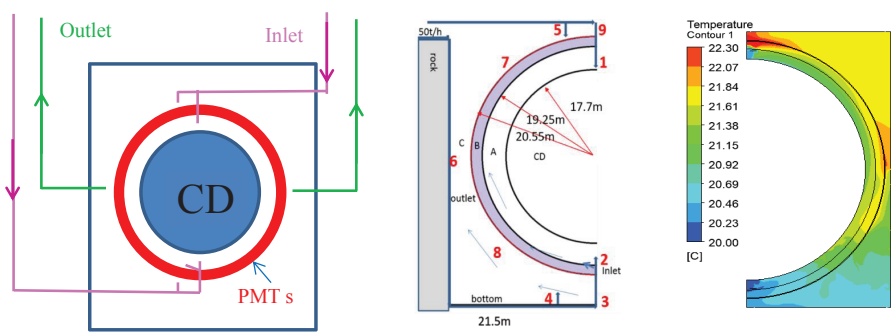

Figure 7: Left: waterway sketch. Middle: layout of water path, $21^{\circ} \mathrm{C}$ water inlet at $1,2,4$ and 5 . Right: temperature distribution, keep the acrylic sphere at $(21 \pm 1)^{\circ} \mathrm{C}$.

\section{Other subsystems of veto}

\subsection{Top tracker bridge}

A long span bridge has to be built over the pool with diameter of $43.5 \mathrm{~m}$. This support structure has following functions: 1) support the top tracker detectors; 2) support the calibration equipments; 3) support the central detector chimney; 4) support partial pipelines of water and liquid scintillator; 5) support pool cover; 6) provide personnel access to the centre. Considering the height limit of the underground space and the structure stiffness, the carbon steel bridge is designed as a box structure with length of $48 \mathrm{~m}$ and width of $25 \mathrm{~m}$, supporting a load about 470 ton. Detailed structure is showed in Figure 8.

\subsection{Earth magnetic field shielding}

The horizontal component of the geomagnetic field is about $40 \mu \mathrm{T}$ and vertical component is about $24 \mu \mathrm{T}$ at JUNO experimental site. Since JUNO detector will use 20 inch PMTs as the 

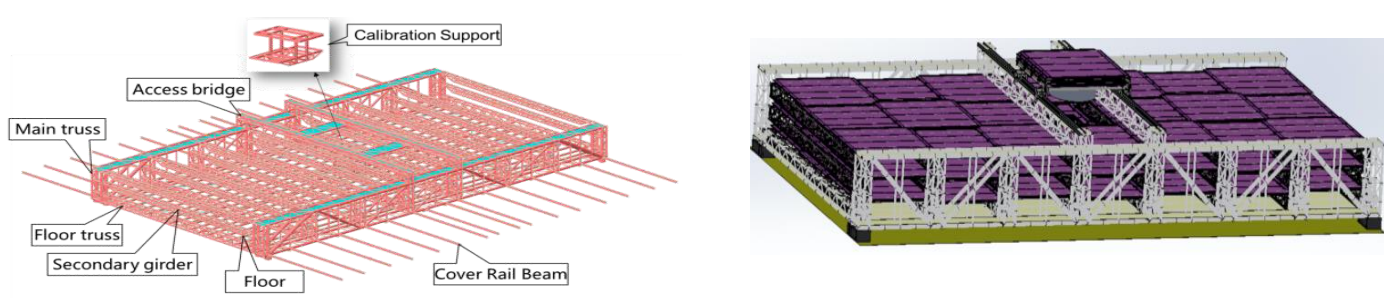

Figure 8: Left: bridge structure. Right: bridge with TT.

main device to accept signal, the geomagnetic field will have a big effect on such large size PMTs. Figure 9 shows the relationship between the PMT collection efficiency and the external magnetic field intensity. A geomagnetic field shielding system consisting of 16 pairs shielding coils will be set up to generate a magnetic field opposite to the direction of geomagnetic field, so as to compensate the affection from earth magnetic field. In this case, the residual field intensity could be reduced to be below $5 \mu \mathrm{T}$ in a spherical region with a radius of $39.5 \mathrm{~m}$. The compensation coil arrangement is also shown in Figure 9.
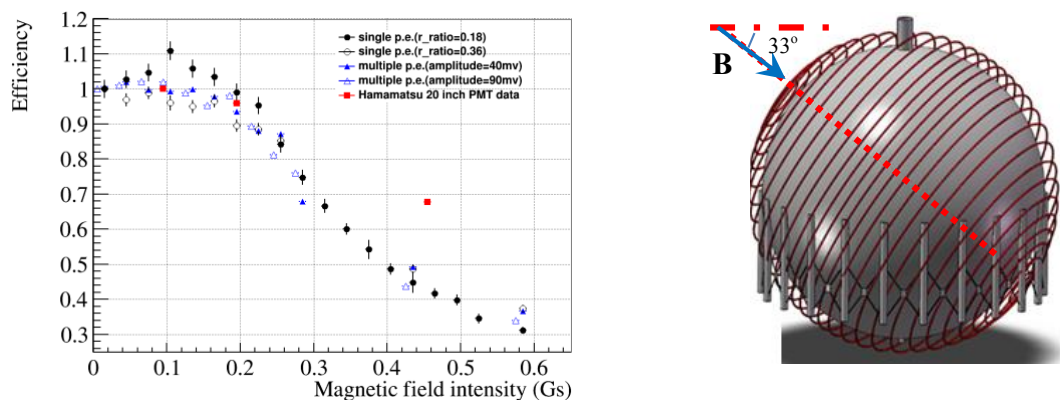

Figure 9: Left: the PMT collection efficiency vs magnetic field intensity. Right: Earth magnetic fieldshielding coils.

\section{Summary}

The main goal of JUNO is to determine the neutrino mass hierarchy. It also has a potential detection for solar neutrinos, geo-neutrinos, supernova neutrinos and the diffuse supernova neutrinos. JUNO detector is being constructed smoothly according to the schedule. All parts of veto detector have finished design and entered manufacturing stage, they are top tracker and bridge, pool liner, cover, PMTs, Tyvek reflection film, veto main support structure, Earth magnetic field shielding, pure water and Radon removal system. Let's look forward to start data taking in 2021.

\section{Acknowledgments}

This work is supported by the Strategic Priority Research Program of the Chinese Academy of Sciences (Grant No. XDA10011200) and the National Natural Science Foundation of China (Grant Nos. 11675203 and 111187528049$)$. 


\section{References}

[1] F. P. An et al. (JUNO Collaboration), Neutrino physics with JUNO, Journal of Physics G: Nuclear and Particle Physics 43 (2016) 030401 (188pp)

[2] F. P. An et al. (JUNO Collaboration), JUNO Conceptual Design Report, 2015, [arXiv:submit/1361070] 